\title{
Abstract Theorems on Exchange of Limits and Preservation of (Semi)continuity of Functions and Measures in the Filter Convergence Setting
}

\author{
Antonio Boccuto ${ }^{1}$ and Xenofon Dimitriou ${ }^{2}$ \\ ${ }^{1}$ Dipartimento di Matematica e Informatica, Via Vanvitelli 1, 06123 Perugia, Italy \\ ${ }^{2}$ Department of Mathematics, University of Athens, Panepistimiopolis, 15784 Athens, Greece
}

Correspondence should be addressed to Antonio Boccuto; antonio.boccuto@unipg.it

Received 28 April 2016; Accepted 7 June 2016

Academic Editor: Ioan Rasa

Copyright (C) 2016 A. Boccuto and X. Dimitriou. This is an open access article distributed under the Creative Commons Attribution License, which permits unrestricted use, distribution, and reproduction in any medium, provided the original work is properly cited.

\begin{abstract}
We give necessary and sufficient conditions for exchange of limits of double-indexed families, taking values in sets endowed with an abstract structure of convergence, and for preservation of continuity or semicontinuity of the limit family, with respect to filter convergence. As a consequence, we give some filter limit theorems and some characterization of continuity and semicontinuity of the limit of a pointwise convergent family of set functions. Furthermore, we pose some open problems.
\end{abstract}

\section{Introduction}

A widely investigated problem in convergence theory and topology is to find necessary and/or sufficient conditions for continuity and/or semicontinuity of the limit of a pointwise convergent net of functions or measures. There have been many recent related studies in abstract structures, like topological spaces, lattice groups, metric semigroups, and cone metric spaces, with respect to usual, statistical, or filter/ideal convergence and associated with the notions of equicontinuity, filter exhaustiveness, and filter continuous convergence (see also [1-9]). The study of semicontinuous functions is associated with quasimetric spaces, that is, spaces endowed with an asymmetric distance function (for a related literature, see, e.g., $[3-5,10-13])$.

A concept associated with these topics is that of strong uniform continuity, which is used to study the problem of finding a topology with respect to which the set of the continuous functions is closed, and pointwise convergence of continuous functions implies convergence in this topology (see also $[1,14,15])$.

Another related field is the study of convergence theorems for measures taking values in abstract structures. When dealing with the classical convergence, it is possible to prove $\sigma$-additivity, (s)-boundedness, and absolute continuity of the limit measure directly from pointwise convergence (with respect to a single order sequence of regulator) of the involved measures, without requiring additional hypotheses. This is not always true in the setting of filter convergence. A historical comprehensive overview, together with a survey on the most recent results and developments, can be found in [16] (see also its bibliography).

In this paper we present a unified axiomatic approach and extend results of this kind to double-indexed families, taking values in abstract structures, whose particular cases are lattice groups, topological groups, (quasi)metric semigroups, and cone (quasi)metric spaces. To include both continuity and semicontinuity, we assume the existence of a "generalized distance" function, which is assumed to satisfy only the triangular property and takes values in a group endowed with a suitable system of "intervals" or "half lines" containing its neutral element 0 . Thus, both topological groups and lattice groups endowed with $(r)-,(D)$-, or order convergence are particular cases of these abstract structures. We prove some results on exchange of limits in the setting of filter convergence. Observe that the involved "distance" can be symmetric or asymmetric (for a literature, see also $[3,5,10]$ and their bibliographies). Furthermore, in our setting, both sequences and nets of functions/measures are included, and note that it is possible 
to consider them as families endowed with filters (see also [17-19]).

As applications, we give some necessary and sufficient conditions for continuity from above/below and absolute continuity and semicontinuity of the limit measure in the context of filter convergence, which include the cases of $\sigma$ additivity and (s)-boundedness, showing, by means of related examples, that they are not always satisfied, differently from the classical case. For a literature on measures satisfying upper/lower semicontinuity conditions or similar properties and related applications, see, for instance, [20] and the bibliography therein. Finally, we pose some open problems.

\section{Assumptions and Examples}

We begin with giving our axiomatic approach, which deals with abstract convergence with respect to filters, without using necessarily nets. For a literature about these topics, see, for instance, $[16,17,19,21-24]$ and their bibliographies.

Definition 1. (a) Let $\Lambda$ be any nonempty set, and let $\mathscr{P}(\Lambda)$ be the class of all subsets of $\Lambda$. A family of sets $\mathscr{F} \subset \mathscr{P}(\Lambda)$ is called a filter of $\Lambda$ iff $\mathscr{F} \neq \emptyset, \emptyset \notin \mathscr{F}$, and $A \cap B \in \mathscr{F}$ for each $A, B \in \mathscr{F}$, and $B \in \mathscr{F}$ whenever $B \supset A$ and $A \in \mathscr{F}$.

Some examples are the filter $\mathscr{F}_{\text {cofin }}$ of all subsets of $\mathbb{N}$ whose complement is finite and the filter $\mathscr{F}_{\text {st }}$ of all subsets of $\mathbb{N}$ having asymptotic density one. Some other classes of filters can be found in [16].

(b) Let $R$ be a nonempty set, and let $Y=(Y,+)$ be an abelian group with neutral element 0 . Given $k \in \mathbb{N}$ and $U_{1}$, $U_{2}, \ldots, U_{k} \subset Y$, put $U_{1}+U_{2}+\cdots+U_{k}:=\left\{u_{1}+u_{2}+\cdots+u_{k}\right.$ : $\left.u_{j} \in U_{j}, j=1,2, \ldots, k\right\}$, and $k U:=U+\cdots+U$ ( $k$ times).

(c) Let $\Pi$ be a nonempty set. A $\Pi$-system $\mathcal{U}$ is a class of families $\mathbf{U}=\left(U_{\pi}\right)_{\pi \in \Pi}$ of subsets of $Y$, with $0 \in \bigcap_{\pi \in \Pi} U_{\pi}$ for each $\mathbf{U}=\left(U_{\pi}\right)_{\pi \in \Pi}$, such that for every $\mathbf{U}=\left(U_{\pi}\right)_{\pi \in \Pi}$ and $\mathbf{V}=$ $\left(V_{\pi}\right)_{\pi \in \Pi} \in \mathcal{U}$ there is $\mathbf{W}=\left(W_{\pi}\right)_{\pi \in \Pi} \in \mathcal{U}$ such that $U_{\pi}+V_{\pi} \subset$ $W_{\pi}$ for every $\pi \in \Pi$. Let $\rho: R \times R \rightarrow Y$ be a function, and suppose that

( $\mathscr{H} 1)$ for every $\mathbf{U}=\left(U_{\pi}\right)_{\pi}, \mathbf{V}=\left(V_{\pi}\right)_{\pi} \in \mathcal{U}$ and for each $\pi \in \Pi$ and $a, b, c \in R$, if $\rho(a, b) \in U_{\pi}$ and $\rho(b, c) \in V_{\pi}$, then $\rho(a, c) \in U_{\pi}+V_{\pi}$.

(d) Fix a $\Pi$-system $\mathcal{U}$ on $Y$ and a filter $\mathscr{F}$ of $\Lambda$. A family $b_{\lambda}, \lambda \in \Lambda$, of elements of $R$ is said to $(\mathcal{U} \mathscr{F})$-backward (resp., (UFF)-forward) converge to $b \in R$ iff there is a family $\left(U_{\pi}\right)_{\pi \in \Pi} \in \mathcal{U}$, such that for every $\pi \in \Pi$ there is a set $F \in \mathscr{F}$ with $\rho\left(b_{\lambda}, b\right) \in U_{\pi}$ (resp., $\rho\left(b, b_{\lambda}\right) \in U_{\pi}$ ) for any $\lambda \in F$. We say that $\left(b_{\lambda}\right)_{\lambda}(\mathcal{U} \mathscr{F})$-converges to $b \in R$ iff it $(\mathscr{U} \mathscr{F})$-converges both backward and forward to $b$, and in this case we write $(\mathcal{H} \mathscr{F}) \lim _{\lambda \in \Lambda} b_{\lambda}=b$.

(e) Let $\Xi$ be a nonempty set. Given two families $\left(a_{\lambda, \xi}\right)_{\lambda \in \Lambda, \xi \in \Xi}$ and $\left(a_{\xi}\right)_{\xi \in \Xi}$ of elements of $R$, we say that

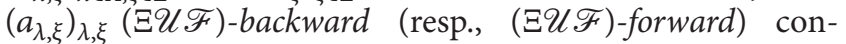
verges to $\left(a_{\xi}\right)_{\xi}$ iff there is a family $\left(U_{\pi}\right)_{\pi \in \Pi} \in \mathcal{U}$, such that for each $\pi \in \Pi$ and $\xi \in \Xi$ there is $F \in \mathscr{F}$ with $\rho\left(a_{\lambda, \xi}, a_{\xi}\right) \in U_{\pi}$ (resp., $\rho\left(a_{\xi}, a_{\lambda, \xi}\right) \in U_{\pi}$ ) for any $\lambda \in F$. Analogously as above it is possible to formulate the notions of $(\Xi \mathscr{U} \mathscr{F})$-convergence and $(\Xi \mathscr{U} \mathscr{F})$-limit.
Remark 2. Observe that, in our context, we will consider filters without dealing explicitly with nets, and this is not a restriction. A net on $R$ is a function $\mathcal{N}: \Lambda \rightarrow R$, where $\Lambda=(\Lambda, \geq)$ is a directed set, namely, a partially ordered set such that for any $\lambda_{1}, \lambda_{2} \in \Lambda$ there exists $\lambda_{0} \in \Lambda$ with $\lambda_{0} \geq \lambda_{j}$, $j=1,2$. Given a directed set $(\Lambda, \geq)$, it is possible to associate the filter $\mathscr{F}_{\Lambda}$ generated by the family $\mathscr{C}^{\prime}:=\left\{\left\{\lambda^{\prime} \in \Lambda: \lambda^{\prime} \geq\right.\right.$ $\lambda\}: \lambda \in \Lambda\}$. Note that $\mathscr{C}^{\prime}$ is a filter base of $\Lambda$; that is, for every $A, B \in \mathscr{C}^{\prime}$ there is an element $C \in \mathscr{C}^{\prime}$ with $C \subset A \cap B$. The filter generated by a filter base $\mathscr{C}$ is the family $\{A \subset \Lambda$ : there is $B \in$ $\mathscr{C}$ with $B \subset A\}$. Conversely, given a filter base $\mathscr{C}:=\left\{C_{\lambda}: \lambda \in\right.$ $\Lambda$, it is possible to associate a directed partial order $\geq$ on $\Lambda$, by setting $\lambda_{1} \geq \lambda_{2}$ if and only if $C_{\lambda_{1}} \subset C_{\lambda_{2}}, \lambda_{1}, \lambda_{2} \in \Lambda$ (see also $[18,19])$.

Example 3. We now present some kinds of abstract space in which our approach can be applied, including both symmetric and asymmetric distance functions (for a literature, see also [3, 5, 10-13]).

(a) Let $R$ be a Dedekind complete lattice group, $Y=R$, and let $\rho(a, b):=|a-b|, a, b \in R$, be the absolute value of $a-b$. It is possible to define different kinds of convergences, as follows (see also [16]).

Let $\Pi_{1}:=\mathbb{R}^{+}$be endowed with the usual order, $\mathcal{U}_{1}:=\left\{([-\varepsilon u, \varepsilon u])_{\varepsilon \in \mathbb{R}^{+}}: u \in R, u>0\right\}((r)-$ convergence); let $\Pi_{2}:=\mathbb{N}$ be with the usual order, $\mathcal{U}_{2}:=$ $\left\{\left(\left[-\sigma_{p}, \sigma_{p}\right]\right)_{p \in \mathbb{N}}:\left(\sigma_{p}\right)_{p}\right.$ is an $(O)$-sequence $\}$, where an $(O)$ sequence is a decreasing sequence in $R$ whose infimum is equal to 0 (order convergence of $(O)$-convergence); let $\Pi_{3}:=\mathbb{N}^{\mathbb{N}}$ be directed with the pointwise order, $\mathcal{U}_{3}:=$ $\left\{\left(\left[-\bigvee_{t=1}^{\infty} a_{t, \varphi(t)}, \bigvee_{t=1}^{\infty} a_{t, \varphi(t)}\right]\right)_{\varphi \in \mathbb{N}^{\mathbb{N}}}:\left(a_{t, l}\right)_{t, l}\right.$ is a $(D)$-sequence $\}$, where a $(D)$-sequence or regulator is a bounded double sequence in $R$ such that $\left(a_{t, l}\right)_{l}$ is an $(O)$-sequence for each $t \in \mathbb{N}((D)$-convergence). The $(D)$-convergence was presented in [25] to give direct proofs of extension theorems for vector lattice-valued functionals and replaces the $\varepsilon$-technique in dealing with suprema and infima of lattice group- or vector lattice-valued families. For technical reasons, sometimes the $(D)$-convergence is easier to handle than $(O)$-convergence, and in particular it is very useful when one replaces a sequence of regulators with a single $(D)$-sequence (for a literature about these topics, see also [16, 23, 26, 27]).

It is not difficult to check that $\mathscr{U}_{j}, j=1,2,3$, are $\Pi_{j}$ systems, satisfying $(\mathscr{H} 1)$.

(b) We can extend the examples given in (a) to the case in which $R$ is a cone metric space (with respect to $Y$ ); that is, $R$ is a nonempty set and $(Y,+)$ is a Dedekind complete lattice group endowed with a distance function $\rho: R \times R \rightarrow Y$, satisfying the following axioms:

(i) $\rho(a, b) \geq 0$ and $\rho(a, b)=0$ if and only if $a=b$.

(ii) $\rho(a, b)=\rho(b, a)$ (symmetric property).

(iii) $\rho(a, c) \leq \rho(a, b)+\rho(b, c)$ (triangular property) for every $a, b, c \in R$.

(See also $[6,28]$.) When a cone metric space $R$ is a semigroup, we say that $R$ is a cone metric semigroup, a cone metric semigroup in which $Y=\mathbb{R}$ is said to be a metric semigroup. Note that the set of fuzzy numbers is a metric semigroup, but 
not a group (see also [20]). If $\rho$ satisfies the first and the third of the above axioms, but not the symmetric property, then we say that $\rho$ is an asymmetric distance function and that $R$ is a cone asymmetric metric space or cone quasimetric space (see also $[3,5,10])$. For example, let $\mathscr{T}$ be a nonempty set, $R=\{f: \mathscr{T} \rightarrow \mathbb{R}, f$ is bounded $\}$, and let $a_{0} \neq 1$ be a fixed positive real number and let $u$ be a fixed element of $R$ with $u>0$. For each $f_{1}, f_{2} \in R$ and $t \in \mathscr{T}$, set

$$
\begin{aligned}
& d_{a_{0}, t}^{(u)}\left(f_{1}(t), f_{2}(t)\right) \\
& \quad= \begin{cases}\left(f_{2}(t)-f_{1}(t)\right) u, & \text { if } f_{1}(t) \leq f_{2}(t), \\
a_{0}\left(f_{1}(t)-f_{2}(t)\right) u, & \text { if } f_{1}(t)>f_{2}(t),\end{cases}
\end{aligned}
$$

and let $\rho_{a_{0}}^{(u)}\left(f_{1}, f_{2}\right)=\bigvee_{t \in \mathscr{T}} d_{a_{0}, t}^{(u)}\left(f_{1}(t), f_{2}(t)\right)$. It is not difficult to see that $\rho_{a_{0}}$ is an asymmetric distance function (see also $[3,10])$.

(c) When $R$ is a lattice group and $Y=R$, it is advisable to deal not only with continuity, but also with upper or lower semicontinuity (see also [4]). In this setting we take $\rho(a, b):=$ $b-a, a, b \in R, \Pi_{j}, j=1,2,3$, as in (a), and $\mathcal{U}_{1}^{(0)}:=\{(\{r \in$ $\left.R: r \leq \varepsilon u\})_{\varepsilon \in \mathbb{R}^{+}}: u \in R, u>0\right\} ; \mathcal{U}_{2}^{(0)}:=\{(\{r \in R: r \leq$ $\left.\left.\sigma_{p}\right\}\right)_{p \in \mathbb{N}}:\left(\sigma_{p}\right)_{p}$ is an (O)-sequence $\} ; \mathscr{U}_{3}^{(0)}:=\{(\{r \in R: r \leq$ $\left.\left.\vee_{t=1}^{\infty} a_{t, \varphi(t)}\right\}\right)_{\varphi \in \mathbb{N}^{N}}:\left(a_{t, l}\right)_{t, l}$ is a $(D)$-sequence $\}$.

(d) Let $R$ be a Hausdorff topological group with neutral element 0 satisfying the first axiom of countability, $Y=R$, $\Pi^{*}=\mathbb{N}, \mathcal{U}^{*}:=\left\{\left(U_{p}\right)_{p \in \mathbb{N}}:\left(U_{p}\right)_{p \in \mathbb{N}}\right.$ is a base of closed symmetric neighborhoods of 0$\}$, and $\rho(a, b)=b-a$. It is not difficult to see that $\mathcal{U}^{*}$ is a $\Pi^{*}$-system (see also $[16,29]$ ).

(e) Let $\mathscr{F}$ be a filter of $\Lambda$. When we consider $(r)$ convergence and $R$ is a cone quasimetric space, a family $\left(b_{\lambda}\right)_{\lambda}$ of elements of $R$ is said to $(r \mathscr{F})$-backward converge to $b$ iff there is $u \in Y, u>0$, with $\left\{\lambda \in \Lambda: \rho\left(b_{\lambda}, b\right) \leq \varepsilon u\right\} \in \mathscr{F}$ for all $\varepsilon>0$. When we deal with $(O)$-sequences, we say that $\left(b_{\lambda}\right)_{\lambda}(O \mathscr{F})$-backward converges to $b$ iff there exists an $(O)$ sequence $\left(\sigma_{p}\right)_{p}$ in $Y$ with $\left\{\lambda \in \Lambda: \rho\left(b_{\lambda}, b\right) \leq \sigma_{p}\right\} \in \mathscr{F}$ for every $p \in \mathbb{N}$. When we consider $(D)$-sequences, we say that the net $\left(b_{\lambda}\right)_{\lambda}(D \mathscr{F})$-backward converges to $b$ iff there exists a regulator $\left(a_{t, l}\right)_{t, l}$ in $Y$ with

$$
\left\{\lambda \in \Lambda: \rho\left(b_{\lambda}, b\right) \leq \bigvee_{t=1}^{\infty} a_{t, \varphi(t)}\right\} \in \mathscr{F}
$$

for each $\varphi \in \mathbb{N}^{\mathbb{N}}$.

When $\Lambda=\mathbb{N}$ and $\mathscr{F}=\mathscr{F}_{\text {cofin }}$, we have the classical $(r)$-, $(O)$-, and $(D)$-(backward, forward) convergence (see also $[3,16]$ ). If $(R,+)$ is a Hausdorff topological group and $Y=R$, then we say that a net $b_{\lambda}, \lambda \in \Lambda$, in $R, \mathscr{F}$-backward converges to $b \in R$ iff $\left\{\lambda \in \Lambda: b_{\lambda}-b \in U\right\} \in \mathscr{F}$ for each neighborhood $U$ of 0 . Similarly as above it is possible to formulate the corresponding notions of $(r \mathscr{F})_{-},(O \mathscr{F})-$, and $(D \mathscr{F})$ (forward) convergences and limits.

(f) When $R$ is a Dedekind complete lattice group, $\left(a_{\lambda, \xi}\right)_{\lambda \in \Lambda, \xi \in \Xi}$ and $\left(a_{\xi}\right)_{\xi \in \Xi}$ are two families in $R$ and $\mathcal{U}$ is the $\Pi$-system associated with $(r)$-convergence (resp., (O)-convergence, (D)-convergence); we say that
$(\Xi r \mathscr{F}) \lim _{\lambda \in \Lambda} a_{\lambda, \xi}=a_{\xi}$ (resp., $(\Xi O \mathscr{F}) \lim _{\lambda \in \Lambda} a_{\lambda, \xi}=a_{\xi}$, $\left.(\Xi D \mathscr{F}) \lim _{\lambda \in \Lambda} a_{\lambda, \xi}=a_{\xi}\right)$ iff $(\Xi \mathscr{U} \mathscr{F}) \lim _{\lambda \in \Lambda} a_{\lambda, \xi}=a_{\xi}$. Analogously it is possible to formulate the corresponding concepts of backward and forward convergences (see also $[3,5,10]$ ). In particular, when $R=\mathbb{R}$ endowed with the usual convergence, since it coincides with $(r)$ - $(O)$-, and $(D)$-convergence, we will denote by $(\mathscr{F})$ - and $(\Xi \mathscr{F})$-(backward, forward) convergence the usual filter (backward, forward) convergence and the ordinary pointwise filter (backward, forward) convergence. When $R$ is a Hausdorff topological group, $\mathscr{U}^{*}, \Pi^{*}$ are as in $(\mathrm{d})$, and we get that the $\left(\Xi \mathcal{U}^{*} \mathscr{F}\right)$-convergence is equivalent to the pointwise $(\mathscr{F})$-convergence, and hence we write $(\mathscr{F}) \lim _{\lambda \in \Lambda} a_{\lambda, \xi}=a_{\xi}$ for every $\xi \in \Xi$, or $(\Xi \mathscr{F}) \lim _{\lambda \in \Lambda} a_{\lambda, \xi}=a_{\xi}$.

(g) Observe that, in general, a family $\left(b_{\lambda}\right)_{\lambda}$ can be backward (resp., forward) convergent to more than one element. For example, if $R$ is a Dedekind complete lattice group, $\Lambda$ is a nonempty set, $\mathscr{F}$ is any filter of $\Lambda, \rho(a, b)=b-a$ for every $a, b \in R, b_{\lambda}=0$ for every $\lambda \in \Lambda$, and $b$ is any element of $R$ with $b \leq 0$ (resp., $b \geq 0$ ), then it is not difficult to see that $\left(b_{\lambda}\right)_{\lambda}(r \mathscr{F})$-backward (resp., $(r \mathscr{F})$-forward) converges to $b$.

(h) In general, backward and forward convergence are not equivalent. For example, similarly as in (1), let $\mathscr{T}$ be a nonempty set, let $\Lambda:=[1,+\infty[$ be endowed with the usual order, let $\mathscr{F}$ be a filter of $\Lambda$ containing all half lines $[c,+\infty[$ with $c \geq 1$, pick $R=\{f: \mathscr{T} \rightarrow \mathbb{R}, f$ is bounded $\}$, and let $\mathbf{0}, \mathbf{1}$ be those functions which associate with every element of $\mathscr{T}$ the real constants 0,1 , respectively. For any $f_{1}, f_{2} \in R$ and $t \in \mathscr{T}$, set

$$
\begin{aligned}
d_{t}^{\prime} & \left(f_{1}(t), f_{2}(t)\right) \\
& = \begin{cases}\left(f_{2}(t)-f_{1}(t)\right) \cdot \mathbf{1}, & \text { if } f_{1}(t) \leq f_{2}(t), \\
1, & \text { if } f_{1}(t)>f_{2}(t),\end{cases}
\end{aligned}
$$

and put $\rho^{\prime}\left(f_{1}, f_{2}\right):=\bigvee_{t \in \mathscr{T}} d_{t}^{\prime}\left(f_{1}(t), f_{2}(t)\right)$. It is not difficult to check that $\rho^{\prime}$ is an asymmetric distance function (see also $[3,10])$. For each $\lambda \in \Lambda$, set $f_{\lambda}:=1 / \lambda \cdot \mathbf{1}$ and $h_{\lambda}:=$ $-f_{\lambda}=-1 / \lambda \cdot \mathbf{1}$. Note that $d\left(\mathbf{0}, f_{\lambda}\right)=f_{\lambda}, d\left(f_{\lambda}, \mathbf{0}\right)=\mathbf{1}$, $d\left(h_{\lambda}, \mathbf{0}\right)=f_{\lambda}$, and $d\left(\mathbf{0}, h_{\lambda}\right)=\mathbf{1}$. From this it is not difficult to deduce that the family $\left(f_{\lambda}\right)_{\lambda}(r \mathscr{F})$-forward converges to $\mathbf{0}$ and $\left(h_{\lambda}\right)_{\lambda}(r \mathscr{F})$-backward converges to $\mathbf{0}$, while $\left(f_{\lambda}\right)_{\lambda}$ does not $(r \mathscr{F})$-backward converge to $\mathbf{0}$ and $\left(h_{\lambda}\right)_{\lambda}$ does not $(r \mathscr{F})$ forward converge to $\mathbf{0}$.

However, if $\Lambda$ is any nonempty set, $\mathscr{F}$ is any filter of $\Lambda, \rho_{a_{0}}^{(u)}$ is as in (1), and $C_{a_{0}}=\max \left\{a_{0}, 1 / a_{0}\right\}$, then it is not difficult to see that $\rho_{a_{0}}^{(u)}\left(f_{1}, f_{2}\right) \leq C_{a_{0}} \rho_{a_{0}}^{(u)}\left(f_{2}, f_{1}\right)$ whenever $f_{1}, f_{2} \in$ $R$. From this it follows that a family $\left(f_{\lambda}\right)_{\lambda \in \Lambda}$ in $R$ is $(r \mathscr{F})$ backward convergent if and only if it is $(r \mathscr{F})$-forward convergent. We claim that, in this case, the involved limit coincides. Indeed, if $\left(f_{\lambda}\right)_{\lambda}(r \mathscr{F})$-backward converges to $f_{0}$ and $(r \mathscr{F})$ forward converges to $h_{0}$ with respect to $\rho_{a_{0}}^{(u)}$, then there exist $v, w \in R$, such that for every $\varepsilon>0$ there are $F_{1}, F_{2} \in \mathscr{F}$ with $\rho_{a_{0}}^{(u)}\left(h_{0}, f_{\lambda}\right) \leq \varepsilon v$ for every $\lambda \in F_{1}, \rho_{a_{0}}^{(u)}\left(f_{\lambda}, f_{0}\right) \leq \varepsilon w$ whenever $\lambda \in F_{2}$. Note that $F_{1} \cap F_{2} \in \mathscr{F}$. If $\lambda_{0}$ is any fixed element of 
$F_{1} \cap F_{2}$, then from the triangular property of $\rho_{a_{0}}^{(u)}$ we deduce that

$$
\begin{aligned}
\rho_{a_{0}}^{(u)}\left(h_{0}, f_{0}\right) & \leq \rho_{a_{0}}^{(u)}\left(h_{0}, f_{\lambda_{0}}\right)+\rho_{a_{0}}^{(u)}\left(f_{\lambda_{0}}, f_{0}\right) \\
& \leq \varepsilon(v+w) .
\end{aligned}
$$

Thus, by arbitrariness of $\varepsilon$, we get $\rho_{a_{0}}^{(u)}\left(h_{0}, f_{0}\right)=0$, and hence $h_{0}=f_{0}$, getting the claim.

\section{The Main Results}

In this section we give the fundamental results of the paper in our unified setting, which includes lattice groups, cone metric spaces, metric groups and topological groups, symmetric and asymmetric distances, continuity and semicontinuity of the limit, and families of functions and of measures. We first present the notion of weak filter backward and forward exhaustiveness in our abstract context, which extends the corresponding ones given in the literature and the classical concept of equicontinuity (see also $[4,8,16,30]$ ).

Definition 4. (a) Let $\Xi$ be a nonempty set; fix $\xi \in \Xi$ and let $\mathcal{S}_{\xi}$ be a filter of $\Xi$. One says that the family $\left(a_{\lambda, \xi}\right)_{\lambda, \xi}$ is

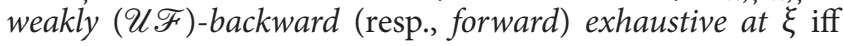
there exists a family $\left(U_{\pi}\right)_{\pi \in \Pi} \in \mathcal{U}$ such that for each $\pi \in \Pi$ there is a set $S \in \mathcal{S}_{\xi}$ such that for every $\zeta \in S$ there is a set $F_{\zeta} \in$ $\mathscr{F}$ with $\rho\left(a_{\lambda, \zeta}, a_{\lambda, \xi}\right) \in U_{\pi}$ (resp., $\left.\rho\left(a_{\lambda, \xi}, a_{\lambda, \zeta}\right) \in U_{\pi}\right)$ for any $\lambda \epsilon$

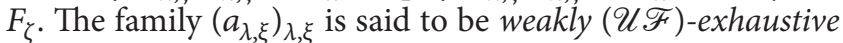
at $\xi$ iff it is both weakly $(\mathscr{U} \mathscr{F})$-backward and weakly $(\mathscr{U} \mathscr{F})$-forward exhaustive at $\xi$.

(b) Let $\mathcal{S}_{\xi}, \xi \in \Xi$, be a family of filters of $\Xi$. One says that $\left(a_{\lambda, \xi}\right)_{\lambda, \xi}$ is weakly $(\mathcal{U} \mathscr{F})$ - (backward, forward) exhaustive on $\Xi$ iff it is weakly ( $\mathscr{U} \mathscr{F})$ - (backward, forward) exhaustive at every $\xi \in \Xi$ with respect to a single family $\mathbf{U} \in \mathcal{U}$, independent of $\xi$.

Example 5. We now show that, in general, weak $(\mathscr{U} \mathscr{F})$ backward and forward exhaustiveness do not coincide. Let $\Lambda=R=\Xi=\mathbb{R}, Y=\mathbb{R}$, be equipped with the usual convergence; that is, let $\Pi:=\mathbb{R}^{+}$be endowed with the usual order, and $\mathcal{U}:=\left\{([-\varepsilon \mathcal{u}, \varepsilon u])_{\varepsilon \in \mathbb{R}^{+}}: u \in \mathbb{R}^{+}\right\}$. Let us define $\rho: \mathbb{R} \times \mathbb{R} \rightarrow \mathbb{R}$ by

$$
\rho(\xi, \zeta):=\left\{\begin{array}{ll}
\zeta-\xi, & \text { if } \xi \leq \zeta, \\
1, & \text { if } \xi>\zeta,
\end{array} \quad \xi, \zeta \in \mathbb{R} .\right.
$$

It is not difficult to see that $\rho$ is an asymmetric distance function (see also [10, Example 5.3]). Let $\mathscr{F}$ be any filter of $\Lambda$, and, for every $\xi \in \Xi$, let $\mathcal{S}_{\xi}$ be the filter of all neighborhoods of $\xi$ with respect to the topology generated by $\rho$. Set $a_{\lambda, \xi}:=$ $\xi+\lambda, \xi, \lambda \in \mathbb{R}$. We claim that the family $\left(a_{\lambda, \xi}\right)_{\lambda, \xi}$ is weakly $(\mathcal{U} \mathscr{F})$-forward exhaustive at $\xi$. Indeed, in correspondence with $\varepsilon>0$, take $\eta:=\min \{\varepsilon, 1 / 2\}$, and set $F_{\zeta}:=\Lambda$ for any $\zeta \in[\xi, \xi+\eta]=S_{\rho}(\xi, \eta)$, where $S_{\rho}(\xi, \eta)$ denotes the ball of center $\xi$ and radius $\eta$ with respect to $\rho$. For every $\lambda \in \Lambda$ and $\zeta \in[\xi, \xi+\eta]$ we get $\rho\left(a_{\lambda, \xi}, a_{\lambda, \zeta}\right)=\zeta+\lambda-(\xi+\lambda)=\zeta-\xi \in[-\eta, \eta]$, getting the claim.
Now, in correspondence with every $\xi \in \mathbb{R}$ and $\theta>0$, let $\eta=\min \{\theta, 1\}$ and take $\zeta=\xi+\eta$. Note that $\zeta \in$ $S_{\rho}(\xi, \theta)$. Choose arbitrarily $F \in \mathscr{F}$. It is not hard to see that $\rho\left(a_{\lambda, \zeta}, a_{\lambda, \xi}\right)=\rho\left(a_{\lambda, \xi+\eta}, a_{\lambda, \xi}\right)=1$ for every $\lambda \in F$. Hence, the family $\left(a_{\lambda, \xi}\right)_{\lambda, \xi}$ is not weakly $(\mathcal{U} \mathscr{F})$-backward exhaustive at $\xi$. Furthermore note that, analogously as in (3), it is not difficult

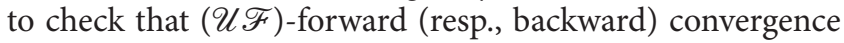
does not imply $(\mathcal{U} \mathscr{F})$-backward (resp., forward) convergence with respect to $\rho$.

The following result deals with characterizations and properties of the limit family and extends [3, Theorem 3.1], $[4$, Theorems 2.5, 2.6], and [6, Theorem 3.1] to the abstract context.

Theorem 6. Assume that $\left(a_{\lambda, \xi}\right)_{\lambda, \xi}(\Xi \mathscr{U} \mathscr{F})$-converges to $\left(a_{\xi}\right)_{\xi}$, fix $\xi \in \Xi$, and let $\mathcal{S}_{\xi}$ be a filter of $\Xi$. Then the following are equivalent:

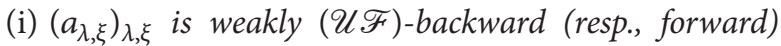
exhaustive at $\xi$.

(ii) $\left(a_{\zeta}\right)_{\zeta}\left(\mathscr{U} \mathcal{S}_{\xi}\right)$-backward (resp., forward) converges to $a_{\xi}$ as $\zeta \rightarrow \xi$.

Proof. We give the proof only in the "backward" case, since the other case is analogous.

(i) $\Rightarrow$ (ii) Let $\left(U_{\pi}\right)_{\pi \in \Pi} \in \mathcal{U}$ be a family related to $(\mathcal{U F})$ backward exhaustiveness of $\left(a_{\lambda, \xi}\right)_{\lambda, \xi}$ at $\xi$. By hypothesis, for each $\pi \in \Pi$, there exists a set $S \in \mathcal{S}_{\xi}$, associated with weak $(\mathcal{U} \mathscr{F})$-backward exhaustiveness. Pick arbitrarily $\zeta \in S$. There is a set $F_{1} \in \mathscr{F}$ with $\rho\left(a_{\lambda, \zeta}, a_{\lambda, \xi}\right) \in U_{\pi}$ for any $\lambda \in F_{1}$. Moreover, thanks to $(\Xi \mathscr{U} \mathscr{F})$-convergence, there is a family $\left(U_{\pi}^{*}\right)_{\pi} \in \mathcal{U}$ such that for every $\pi \in \Pi$ there exists $F_{2} \in \mathscr{F}$ with $\rho\left(a_{\zeta}, a_{\lambda, \zeta}\right) \in U_{\pi}^{*}$ and $\rho\left(a_{\lambda, \xi}, a_{\xi}\right) \in U_{\pi}^{*}$ whenever $\lambda \in F_{2}$. From this and $(\mathscr{H} 1)$ it follows that $\rho\left(a_{\zeta}, a_{\xi}\right) \in 2 U_{\pi}^{*}+U_{\pi}$, getting (ii).

(ii) $\Rightarrow$ (i) By hypothesis, there exists a family $\left(U_{\pi}\right)_{\pi} \in \mathscr{U}$ such that for each $\pi \in \Pi$ there is a set $S \in \mathcal{S}_{\xi}$ with

$$
\rho\left(a_{\zeta}, a_{\xi}\right) \in U_{\pi} \quad \text { whenever } \zeta \in S \text {. }
$$

Choose $\zeta \in S$. By $(\Xi \mathscr{U} \mathscr{F})$-convergence of $\left(a_{\lambda, \xi}\right)_{\lambda, \xi}$ to $\left(a_{\xi}\right)_{\xi}$, there is a family $\left(U_{\pi}^{*}\right)_{\pi} \in \mathcal{U}$ such that for every $\pi \in \Pi$ there is a set $F^{*} \in \mathscr{F}$ with

$$
\begin{aligned}
& \rho\left(a_{\xi}, a_{\lambda, \xi}\right) \in U_{\pi}^{*}, \\
& \rho\left(a_{\lambda, \zeta}, a_{\zeta}\right) \in U_{\pi}^{*}
\end{aligned}
$$

for each $\lambda \in F^{*}$. From (6), (7), and ( $\mathscr{H} 1$ ) we get that for every $\pi \in \Pi$ there is $S \in \mathcal{S}_{\xi}$ such that for each $\zeta \in S$ there exists $F^{*} \in \mathscr{F}$ with $\rho\left(a_{\lambda, \zeta}, a_{\lambda, \xi}\right) \in 2 U_{\pi}^{*}+U_{\pi}$ whenever $\lambda \in F^{*}$. Thus the family $\left(a_{\lambda, \xi}\right)_{\lambda, \xi}$ is weakly $(\mathscr{U} \mathscr{F})$-backward exhaustive at $\xi$. This ends the proof.

Remark 7. Observe that Theorem 6 holds also if $(\Xi \mathscr{U} \mathscr{F})$ convergence is replaced by $(\Xi \mathscr{U} \mathscr{F})$-forward convergence, under the hypothesis that forward convergence implies backward convergence (see also [10]). In general this last condition is essential. Indeed, let $\Lambda:=[1,+\infty[$ be endowed with the 
usual order, let $\mathscr{F}$ be a filter of $\Lambda$ containing all half lines $[c,+\infty[$ with $c \geq 1$, let $\Xi:=[0,1]$ be equipped with the usual distance, let $\mathcal{S}_{\xi}, \xi \in \Xi$, be the filter of all neighborhoods of $\xi$, let $Y=\mathbb{R}$ be endowed with the usual convergence, $R=[0,1] \times[0,1]$, and let $\rho^{*}: R \times R \rightarrow \mathbb{R}$ be defined by

$$
\begin{aligned}
& \rho^{*}\left(\left(\xi_{1}, \xi_{2}\right),\left(\zeta_{1}, \zeta_{2}\right)\right) \\
& = \begin{cases}0, & \text { if }\left(\xi_{1}, \xi_{2}\right)=\left(\zeta_{1}, \zeta_{2}\right), \\
\max \left\{\left|\xi_{1}-\zeta_{1}\right|,\left|\xi_{2}-\zeta_{2}\right|\right\}, & \text { if } \xi_{1} \leq \zeta_{1}, \zeta_{1}>0, \\
1, & \text { otherwise. }\end{cases}
\end{aligned}
$$

It is not difficult to check that $\rho^{*}$ is an asymmetric distance function. For every $\lambda \in \Lambda$ and $\xi \in \Xi$, set $a_{\lambda, \xi}^{*}:=(1 / \lambda, \xi)$. Observe that $\rho^{*}((0, \xi),(1 / \lambda, \xi))=1 / \lambda$ and $\rho^{*}((1 / \lambda, \xi),(0, \xi))=1$ for every $\lambda \in \Lambda$ and $\xi \epsilon$ $\Xi$. It is not difficult to see that the family $\left(a_{\lambda, \xi}^{*}\right)_{\lambda, \xi}(\Xi \mathscr{U F})$ forward converges to $\left(a_{\xi}^{*}\right)_{\xi \in \Xi}$, where $a_{\xi}^{*}=(0, \xi), \xi \in \Xi$, but does not $(\Xi \mathcal{U} \mathscr{F})$-backward converge. Moreover, since $\rho^{*}((0, \zeta),(0,0))=\rho^{*}((0,0),(0, \zeta))=1$, for every $\zeta \in \Xi, \zeta \neq 0$, the family $\left(a_{\zeta}^{*}\right)_{\zeta \in \Xi}$ is neither $\left(\mathcal{U} \mathcal{S}_{\xi}\right)$-backward nor $\left(\mathcal{U} \mathcal{S}_{\xi}\right)$ forward convergent to $a_{0}^{*}$ as $\zeta \rightarrow 0$. Furthermore, we get

$$
\begin{aligned}
\rho^{*}\left(a_{\lambda, \zeta}^{*}, a_{\lambda, 0}^{*}\right) & =\rho^{*}\left(\left(\frac{1}{\lambda}, \zeta\right),\left(\frac{1}{\lambda}, 0\right)\right)=\zeta \\
& =\rho^{*}\left(\left(\frac{1}{\lambda}, 0\right),\left(\frac{1}{\lambda}, \zeta\right)\right)=\rho^{*}\left(a_{\lambda, 0}^{*}, a_{\lambda, \zeta}^{*}\right)
\end{aligned}
$$

for every $\lambda \in \Lambda$ and $\zeta \in \Xi$. From (9) it is not difficult to deduce that the family $\left(a_{\lambda, \xi}^{*}\right)_{\lambda, \xi}$ is both weakly $(\mathcal{U} \mathscr{F})$-forward and weakly $(\mathcal{U} \mathscr{F})$-backward exhaustive at 0 (see also [3, Example 3.7], [10, Example 5.10]).

We now give some kinds of convergences for families, which are some necessary and sufficient conditions for exchange of limits, which extend to our context some results proved in $[1,2,4,6,9]$ about necessary and sufficient conditions for continuity of the pointwise limit of continuous functions. We extend to our setting the concepts of Arzelà, Alexandroff, and strong uniform convergence given in $[1,15$, 31, 32].

Definition 8. (a) Fix $\xi \in \Xi$, and let $\mathcal{S}_{\xi}$ be a filter of $\Xi$. One says that $\left(a_{\lambda, \xi}\right)_{\lambda, \xi}(\mathscr{U} \mathscr{F})$-forward strongly uniformly converges to

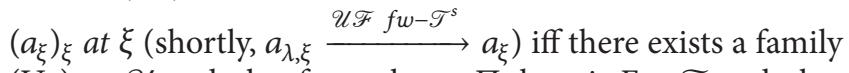
$\left(U_{\pi}\right)_{\pi} \in \mathcal{U}$ such that for each $\pi \in \Pi$ there is $F \in \mathscr{F}$ such that for every $\lambda \in F$ there is a set $S_{\lambda} \in \mathcal{S}_{\xi}$ with $\rho\left(a_{\zeta}, a_{\lambda, \zeta}\right) \in U_{\pi}$ whenever $\zeta \in S_{\lambda}$.

(b) One says that $\left(a_{\lambda, \xi}\right)_{\lambda, \xi}$ is $(\mathcal{U} \mathscr{F})$-forward Arzelà convergent to $\left(a_{\xi}\right)_{\xi}$ at $\xi$ (in brief, $a_{\lambda, \xi} \stackrel{U \mathscr{F} f w-A r z .}{\longrightarrow} a_{\xi}$ ) iff there exists a family $\left(U_{\pi}\right)_{\pi \in \Pi} \in \mathcal{U}$ such that for every $\pi \in \Pi$ and $F \in \mathscr{F}$ there are a finite set $\left\{\lambda_{1}, \lambda_{2}, \ldots, \lambda_{q}\right\} \subset F$ and a set $S \in \mathcal{S}_{\xi}$, such that for each $\zeta \in S$ there is $j \in[1, q]$ with $\rho\left(a_{\zeta}, a_{\lambda_{j}, \zeta}\right) \in U_{\pi}$.

(c) If $\mathcal{S}_{\xi}, \xi \in \Xi$, is a family of filters of $\Xi$, then one says that a finitely uniform cover of $\Xi$ is a family $\mathscr{V}$ of subsets of $\Xi$ such that $\Xi=\bigcup_{V \in \mathscr{V}} V$, and for every $\xi \in \Xi$ there are a set
$S_{\xi} \in \mathcal{S}_{\xi}$ and a finite subset $\mathcal{Y}:=\left\{V_{l_{1}}, \ldots, V_{l_{q}}\right\}$ of $\mathscr{V}$, such that for each $\zeta \in S_{\xi}$ there exists $j \in[1, q]$ with $\zeta \in V_{l_{j}}$.

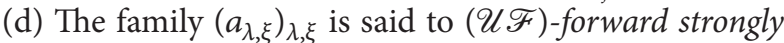
uniformly (resp., $(\mathscr{U} \mathscr{F})$-forward Arzelà) converge to $\left(a_{\xi}\right)_{\xi}$

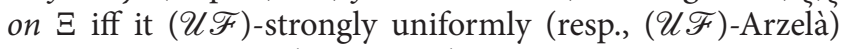
converges to $\left(a_{\xi}\right)_{\xi}$ at $\xi$ for every $\xi \in \Xi$ with respect to a single family $\mathbf{U} \in \mathcal{U}$, independent of $\xi$.

(e) One says that $\left(a_{\lambda, \xi}\right)_{\lambda, \xi}$ is $(\mathcal{U F})$-forward Alexandroff convergent to $\left(a_{\xi}\right)_{\xi}$ on $\Xi$ (shortly, $a_{\lambda, \xi} \stackrel{U \mathscr{F} f w-A l \text {. }}{\longrightarrow} a_{\xi}$ on $\Xi$ ) iff there exists a family $\left(U_{\pi}\right)_{\pi} \in \mathcal{U}$ such that for each $\pi \in \Pi$ and $F \in \mathscr{F}$ there are a nonempty set $\Lambda_{0} \subset F$ and a finitely uniform cover $\left\{V_{\lambda}: \lambda \in \Lambda_{0}\right\}$ of $\Xi$ with $\rho\left(a_{\zeta}, a_{\lambda, \zeta}\right) \in U_{\pi}$ for any $\lambda \in \Lambda_{0}$ and $\zeta \in V_{\lambda}$.

Note that, analogously as above, it is possible to formulate the corresponding concepts of (backward) filter strong uniform, Arzelà, and Alexandroff convergence.

The next result extends [2, Theorem 3.9], [4, Theorems 2.9, 2.11 and Corollary 2.10], and [9, Proposition 3.5].

Theorem 9. Let $\xi \in \Xi$ be fixed, let $\mathcal{S}_{\xi}$ be a filter of $\Xi$, and suppose that

(3.6.1) $\left(\Lambda \mathcal{U} \mathcal{S}_{\xi}\right) \lim _{\zeta \rightarrow \xi} a_{\lambda, \zeta}=a_{\lambda, \xi}$;

(3.6.2) the family $\left(a_{\lambda, \zeta}\right)_{\lambda, \zeta}(\Xi \mathscr{U} \mathscr{F})$-converges to $\left(a_{\zeta}\right)_{\zeta}$.

Then the following are equivalent:

(i) $\left(a_{\zeta}\right)_{\zeta}\left(\mathcal{U} \mathcal{S}_{\xi}\right)$-backward converges to $a_{\xi}$ as $\zeta \rightarrow \xi$.

(ii) $a_{\lambda, \xi} \stackrel{\text { UF } f w-\mathscr{T}^{s}}{\longrightarrow} a_{\xi}$ at $\xi$.

(iii) $a_{\lambda, \xi} \stackrel{\mathscr{U F} f w-A r z .}{\longrightarrow} a_{\xi}$ at $\xi$.

Proof. (i) $\Rightarrow$ (ii) Let $\left(U_{\pi}\right)_{\pi},\left(Y_{\pi}\right)_{\pi}$, and $\left(U_{\pi}^{*}\right)_{\pi} \in \mathcal{U}$ be three families associated with (i), (3.6.1), and (3.6.2), respectively, and take arbitrarily $\pi \in \Pi$. By (3.6.2), there is $F \in \mathscr{F}$ with $\rho\left(a_{\xi}, a_{\lambda, \xi}\right) \in U_{\pi}^{*}$ for all $\lambda \in F$. By (3.6.1) and (i), for each $\lambda \in F$ there is $S_{\lambda} \in \mathcal{S}_{\xi}$ with $\rho\left(a_{\lambda, \xi}, a_{\lambda, \zeta}\right) \in Y_{\pi}$ and $\rho\left(a_{\zeta}, a_{\xi}\right) \in U_{\pi}$ for any $\zeta \in S_{\lambda}$. For such $\zeta$ 's, taking into account ( $\left.\mathscr{H} 1\right)$, we have $\rho\left(a_{\zeta}, a_{\lambda, \zeta}\right) \in Y_{\pi}+U_{\pi}^{*}+U_{\pi}$, getting (ii).

(ii) $\Rightarrow$ (iii) Let $\left(U_{\pi}\right)_{\pi} \in \mathscr{U}$ be a family, according to $(\mathscr{U} \mathscr{F})$ strong uniform convergence. Choose arbitrarily $\pi \in \Pi$ and $F \in \mathscr{F}$, and let $F_{0} \in \mathscr{F}$ be associated with $(\mathcal{U F})$-strong uniform convergence. Pick any finite set $W:=\left\{\lambda_{1}, \ldots, \lambda_{q}\right\} \subset$ $F \cap F_{0} \in \mathscr{F}$ : since $\mathscr{F}$ is a filter, $W$ does exist. For every $j \in[1, q]$, let $S_{\lambda_{j}} \in \mathcal{S}_{\xi}$ be related to $(\mathcal{U} \mathscr{F})$-strong uniform convergence, and set $S=\bigcap_{j=1}^{q} S_{\lambda_{j}}$. Note that $S \in \mathcal{S}_{\xi}$. By construction, for each $\zeta \in S$ and $j \in[1, q]$, we get $\rho\left(a_{\zeta}, a_{\lambda_{j}, \zeta}\right) \in$ $U_{\pi}$. Thus, we obtain (iii).

(iii) $\Rightarrow$ (i) Let $\left(U_{\pi}\right)_{\pi},\left(Y_{\pi}\right)_{\pi}$, and $\left(Z_{\pi}\right)_{\pi} \in \mathcal{U}$ be families related to (iii), (3.6.1), and (3.6.2), respectively. By (3.6.2), there is a set $F \in \mathscr{F}$ with

$$
\rho\left(a_{\lambda, \xi}, a_{\xi}\right) \in Z_{\pi} \quad \forall \lambda \in F
$$


Choose arbitrarily $\pi \in \Pi$. By (iii), in correspondence with $\pi$ and $F$, there exist a finite set $\left\{\lambda_{1}, \lambda_{2}, \ldots, \lambda_{q}\right\} \subset F$ and a set $S \in \mathcal{S}_{\xi}$ such that for every $\zeta \in S_{\xi}$ there is $j \in[1, q]$ with

$$
\rho\left(a_{\zeta}, a_{\lambda_{j}, \zeta}\right) \in U_{\pi}
$$

Thanks to (3.6.1), we find a set $W \in \mathcal{S}_{\xi}$, without loss of generality $W \subset S$, with

$$
\rho\left(a_{\lambda_{j}, \zeta}, a_{\lambda_{j}, \xi}\right) \in Y_{\pi}
$$

for each $\zeta \in W$. From (10), (11), (12), and ( $\mathscr{H} 1)$ it follows that $\rho\left(a_{\zeta}, a_{\xi}\right) \in U_{\pi}+Y_{\pi}+Z_{\pi}$ for every $\zeta \in W_{\xi}$, getting (i).

Remark 10. (a) In general, Theorem 9 does not hold, when the involved "forward" convergences are replaced by the corresponding "backward" ones. Indeed, for example, let $\Lambda:=$ $\mathbb{N}, \mathscr{F}$ be any filter of $\mathbb{N}$, let $\Xi=[0,1]$ be endowed with the usual metric, $\xi=1$, and let $\mathcal{S}_{\xi}$ be the filter of all neighborhoods of 1 contained in $[0,1], R=Y=\mathbb{R}, \mathcal{U}:=$ $\left\{(\{\zeta \in \mathbb{R}: \zeta \leq \varepsilon u\})_{\varepsilon \in \mathbb{R}^{+}}: u \in \mathbb{R}^{+}\right\}, \rho(a, b)=b-a, a, b \in \mathbb{R}$. Put $a_{n, \zeta}:=\zeta^{n}, n \in \mathbb{N}, \zeta \in[0,1]$. We get $\lim _{\zeta \rightarrow \xi} a_{n, \zeta}=1$ for every $n \in \mathbb{N}$, and

$$
a_{\zeta}:=\lim _{n} a_{n, \zeta}= \begin{cases}0, & \text { if } 0 \leq \zeta<1, \\ 1, & \text { if } \zeta=1 .\end{cases}
$$

Note that for each $\varepsilon>0$ and $n \in \mathbb{N}$ we get

$$
\rho\left(a_{n, \zeta}, a_{\zeta}\right)=a_{\zeta}-a_{n, \zeta}= \begin{cases}-\zeta^{n}<\varepsilon, & \text { if } 0 \leq \zeta<1, \\ 0<\varepsilon, & \text { if } \zeta=1 .\end{cases}
$$

Hence, $a_{n, \xi} \stackrel{\text { UF } b w-\mathscr{T}^{s}}{\longrightarrow} a_{\xi}$ at $\xi$. On the other hand, for every $n \in \mathbb{N}$ and for each neighborhood $S$ of 1 contained in $[0,1]$ there is a real number $\zeta \in S \cap] 0,1$ [, close enough to 1 , with $\zeta>1 / 2^{1 / n}$, and hence

$$
\rho\left(a_{\zeta}, a_{n, \zeta}\right)=a_{n, \zeta}-a_{\zeta}=\zeta^{n}>\frac{1}{2} .
$$

Thus, $a_{n, \xi} \stackrel{\mathscr{U F} f w-\mathscr{T}^{s}}{\longrightarrow} a_{\xi}$ at $\xi$. The family $\left(a_{\zeta}\right)_{\zeta}\left(\mathcal{U} \mathcal{S}_{\xi}\right)$-forward, but not backward, converges to $a_{\xi}$ as $\zeta \rightarrow \xi$ : indeed for every $\zeta \in\left[0,1\left[\right.\right.$ we have $\rho\left(a_{\xi}, a_{\zeta}\right)=a_{\zeta}-a_{\xi}=-1<\varepsilon$ for each $\varepsilon>0$, but $\rho\left(a_{\zeta}, a_{\xi}\right)=a_{\xi}-a_{\zeta}=1$. Note that the function $\zeta \mapsto a_{\zeta}, \zeta \in[0,1]$, is upper semicontinuous, but not lower semicontinuous, at 1 .

(b) Observe that Theorem 9 does not hold, where in (3.6.1) the involved convergence is replaced by the corresponding backward or forward convergence (see also [2, Example 3.3]).

Let $\Lambda, \mathscr{F}, R, Y, \mathcal{U}$, and $\rho$ be as in (a), let $\Xi:=\mathbb{R}$ be endowed with the usual metric, $\xi=0$, and let $\mathcal{\delta}_{\xi}$ be the filter of all neighborhoods of 0 . Set

$$
a_{n, \zeta}:= \begin{cases}0, & \text { if } \left.\zeta \in]-\infty,-\frac{1}{n}\right] \cup\{0\} \cup\left[\frac{1}{n},+\infty[,\right. \\ 1, & \text { otherwise. }\end{cases}
$$

Observe that $a_{\zeta}:=\lim _{n} a_{n, \zeta}=0$ for every $\zeta \in \mathbb{R}$, so that (3.6.2) holds, and condition (i) of Theorem 9 is fulfilled. Moreover it is not difficult to see that, for each $n \in \mathbb{N}, a_{n, \zeta}$ converges backward, but not forward, to $a_{n, 0}=0$ as $\zeta$ tends to 0 , and hence (3.6.1) is not verified. However, note that for every $n \epsilon$ $\mathbb{N}$ and for every neighborhood $U$ of 0 there is $\zeta \in U$ with $a_{n, \zeta}=1$, and hence $\rho\left(a_{\zeta}, a_{n, \zeta}\right)=a_{n, \zeta}-a_{\zeta}=1$. Thus, condition (ii) of Theorem 9 is not satisfied.

Furthermore, if we define $b_{n, \zeta}, n \in \mathbb{N}, \zeta \in \mathbb{R}$, by

$$
b_{n, \zeta}:= \begin{cases}1, & \text { if } \left.\zeta \in]-\infty,-\frac{1}{n}\right] \cup\left[\frac{1}{n},+\infty[,\right. \\ 2, & \text { if } \zeta=0, \\ 0, & \text { otherwise, }\end{cases}
$$

then

$$
b_{\zeta}:=\lim _{n} b_{n, \zeta}= \begin{cases}1, & \text { if } \zeta \neq 0 \\ 2, & \text { if } \zeta=0\end{cases}
$$

Hence, (3.6.2) is satisfied, but condition (i) of Theorem 9 does not hold. Observe that, for any $n \in \mathbb{N}, b_{n, \zeta}$ converges forward, but not backward, to $b_{n, 0}=2$ as $\zeta$ tends to 0 , and hence (3.6.1) is not satisfied. On the other hand, since $\rho\left(b_{\zeta}, b_{n, \zeta}\right)=b_{n, \zeta}-$ $b_{\zeta} \leq 0$ for any $n \in \mathbb{N}$ and $\zeta \in \mathbb{R}$, we get that condition (ii) of Theorem 9 is fulfilled.

We now turn to the main theorem in our abstract setting, which extends [1, Theorems 4.7, 4.11], [2, Theorem 3.10], [4, Theorem 2.12], and [6, Corollary 3.5] to our abstract unified setting.

Theorem 11. Let $\mathcal{S}_{\xi}, \xi \in \Xi$, be a family of filters of $\Xi$, with the property that $\xi \in S$ for every $\xi \in \Xi$ and $S \in \mathcal{S}_{\xi}$. Suppose that (3.6.2) holds and that

(3.8.1) $\left(\Lambda \mathscr{U} \mathcal{S}_{\xi}\right) \lim _{\zeta \rightarrow \xi} a_{\lambda, \zeta}=a_{\lambda, \xi}$ for each $\xi \in \Xi$ with respect to a single family $\mathbf{Y} \in \mathcal{U}$, independent of both $\lambda$ and $\xi$.

Then the following are equivalent:

(i) $\left(a_{\zeta}\right)_{\zeta}\left(\mathcal{U} \mathcal{S}_{\xi}\right)$-backward converges to $a_{\xi}$ as $\zeta \rightarrow \xi$ for every $\xi \in \Xi$, with respect to a single family $\mathbf{U} \in \mathcal{U}$, independent of $\xi$.

(ii) $a_{\lambda, \xi} \stackrel{\text { UF } f w-\mathscr{T}^{s}}{\longrightarrow} a_{\xi}$ on $\Xi$.

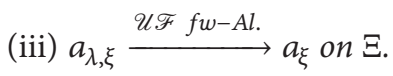

(iv) $a_{\lambda, \xi} \stackrel{\text { UF } f w-A r z .}{\longrightarrow} a_{\xi}$ on $\Xi$.

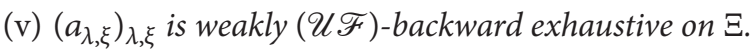

Proof. (i) $\Leftrightarrow$ (v) It is similar to Theorem 6 .

(i) $\Leftrightarrow$ (ii) $\Leftrightarrow$ (iv) It is similar to Theorem 9 .

(ii) $\Rightarrow$ (iii) Let $\left(W_{\pi}\right)_{\pi} \in \mathcal{U}$ be a family associated with $\mathcal{U} \mathscr{F}-\mathscr{T}^{s}$-convergence of $\left(a_{\lambda, \xi}\right)_{\lambda, \xi}$ to $\left(a_{\xi}\right)_{\xi}$. Choose arbitrarily $\pi \in \Pi$ and $F_{0} \in \mathscr{F}$. By (ii), for every $\xi \in \Xi$, there exists a set $F_{\xi} \in \mathscr{F}$, such that for every $\lambda \in F_{\xi}$ there is $S_{\lambda, \xi} \in \mathcal{S}_{\xi}$ with 
$\rho\left(a_{\zeta}, a_{\lambda, \zeta}\right) \in W_{\pi}$ for any $\zeta \in S_{\lambda, \xi}$. Set $F=\bigcup_{\xi \in \Xi} F_{\xi}$ : note that $\Lambda_{0} \in \mathscr{F}$, where $\Lambda_{0}:=F \cap F_{0} \neq \emptyset$. For every $\lambda \in \Lambda_{0}$, let

$$
E_{\lambda}:=\left\{\xi \in \Xi: \rho\left(a_{\zeta}, a_{\lambda, \zeta}\right) \in W_{\pi} \text { for every } \zeta \in S_{\lambda, \xi}\right\} .
$$

Pick arbitrarily $\xi \in \Xi$ and choose $\lambda \in \Lambda_{0}$. We have $\rho\left(a_{\zeta}, a_{\lambda, \zeta}\right) \in W_{\pi}$ whenever $\zeta \in S_{\lambda, \xi}$. Thus, $\Xi=\bigcup_{\lambda \in \Lambda_{0}} E_{\lambda}$. For each $\lambda \in \Lambda_{0}$, set $S_{\lambda}=\bigcup_{\xi \in E_{\lambda}} S_{\lambda, \xi}$. Note that $\mathscr{V}:=\left\{S_{\lambda}\right.$ : $\left.\lambda \in \Lambda_{0}\right\}$ is a cover of $\Xi$. For every $\lambda \in \Lambda_{0}$ and $\zeta \in S_{\lambda}$ there is $\xi \in E_{\lambda}$ with $\zeta \in S_{\lambda, \xi}$, and hence $\rho\left(a_{\zeta}, a_{\lambda, \zeta}\right) \in W_{\pi}$. Now, in correspondence with $\xi \in \Xi$, choose an element $\lambda \in F_{\xi} \cap F_{0}$ and pick $S_{\lambda, \xi}$. Note that $S_{\lambda, \xi} \in \mathcal{S}_{\xi}$ and $S_{\lambda, \xi} \subset S_{\lambda}$. Thus, $\mathscr{V}$ is a finitely uniform cover of $\Xi$, with $S_{\xi}=S_{\lambda, \xi}$ and $\mathscr{Y}=\left\{S_{\lambda}\right\}$. Therefore, $\left(a_{\lambda, \xi}\right)_{\lambda, \xi}(\mathscr{U F})$-Alexandroff converges to $\left(a_{\xi}\right)_{\xi}$.

(iii) $\Rightarrow$ (iv) Let $\left(U_{\pi}\right)_{\pi \in \Pi} \in \mathscr{U}$ be a family associated with (UFF)-Alexandroff convergence of $\left(a_{\lambda, \xi}\right)$ to $\left(a_{\xi}\right)_{\xi}$. Pick arbitrarily $\xi \in \Xi, \pi \in \Pi$, and $F \in \mathscr{F}$. By (iii), there are a nonempty set $\Lambda_{0} \subset F$ and a finitely uniform cover $\mathscr{V}:=\left\{V_{\lambda}: \lambda \in \Lambda_{0}\right\}$ of $\Xi$, with $\rho\left(a_{\zeta}, a_{\lambda, \zeta}\right) \in U_{\pi}$ for each $\lambda \in \Lambda_{0}$ and $\xi \in V_{\lambda}$. Since $\mathscr{V}$ is a finitely uniform cover, in correspondence with $\xi$, there exist a set $S_{\xi} \in \mathcal{S}_{\xi}$ and a finite subset $\left\{\lambda_{1}, \lambda_{2}, \ldots, \lambda_{q}\right\} \subset$ $\Lambda_{0}$, such that for every $\zeta \in S_{\xi}$ there is $j \in[1, q]$ with $\zeta \in V_{\lambda_{j}}$. Thus $\rho\left(a_{\zeta}, a_{\lambda_{j}, \zeta}\right) \in U_{\pi}$, and so we obtain (iv). This ends the proof.

Remark 12. Observe that when the function $\rho$ is symmetric, Theorems 6, 9, and 11 can be viewed as necessary and sufficient conditions in order to have exchange of limits (for a related literature, see also $[16,26,33])$.

\section{Applications to Set Functions}

In this section, as consequences of Theorems 6, 9, and 11, we will give some necessary and sufficient conditions for some kind of continuity and semicontinuity of the limit of set functions. We begin with proving a result on continuity from below the limit measure. Note that, thanks to the limit theorems existing in the literature, these conditions are often fulfilled (for a comprehensive historical survey, see [16] and its bibliography). However, we give an example in which these properties do not hold in the setting of filter convergence.

Let $\Lambda$ be any nonempty set, let $\mathscr{F}$ be any filter of $\Lambda$, let $G$ be any infinite set, let $\Sigma$ be $\sigma$-algebra of subsets of $G$, and let $(R, Y)$ be a (symmetric) cone metric semigroup, $\Xi:=\mathbb{N} \cup$ $\{+\infty\}, \xi:=+\infty, \mathcal{S}_{\xi}:=\left\{F \cup\{+\infty\}: F \in \mathscr{F}_{\text {cofin }}\right\}$. It is not difficult to check that $\mathcal{S}_{\xi}$ is a filter of $\Xi$. Moreover, let $\mathcal{U}$ be a fixed $\Pi$-system associated with $(R, Y)$.

A set function $m: \Sigma \rightarrow R$ is said to be $\mathcal{U}$-continuous from below (resp., from above) on $\Sigma$ iff $\left(\mathcal{U} \mathcal{S}_{\xi}\right) \lim _{k} \rho\left(m\left(C_{k}\right), m(C)\right)=0$ for every increasing (resp., decreasing) sequence $\left(C_{k}\right)_{k}$ in $\Sigma$ whose union (resp., intersection) is equal to $C$. A consequence of Theorems 6 and 9 is the following.

Theorem 13. Let $m_{\lambda}: \Sigma \rightarrow R, \lambda \in \Lambda$, be a family of set functions, $\mathcal{U}$-continuous from below on $\Sigma$, with respect to a family $\mathbf{U} \in \mathcal{U}$ independent of $\lambda$. Suppose that

(4.1.1) $m(E):=(\mathscr{U} \mathscr{F}) \lim _{\lambda} m_{\lambda}(E), E \in \Sigma$, exists in $R$ with respect to a family $\mathbf{V} \in \mathcal{U}$ independent of $E \in \Sigma$.
Then the following are equivalent:

(i) $m$ is $\mathscr{U}$-continuous from below on $\Sigma$.

(ii) For every increasing sequence $\left(C_{k}\right)_{k}$ in $\Sigma$ there is a family $\left(W_{\pi}\right)_{\pi} \in \mathcal{U}$ such that for any $\pi \in \Pi$ there is $\bar{k} \in \mathbb{N}$ such that, for every $k \geq \bar{k}$, there is a set $F \in \mathscr{F}$ with $\rho\left(m_{\lambda}\left(C_{k}\right), m_{\lambda}(C)\right) \in W_{\pi}$ for each $\lambda \in F$.

(iii) For any increasing sequence $\left(C_{k}\right)_{k}$ in $\Sigma$ there is a family $\left(U_{\pi}\right)_{\pi} \in \mathcal{U}$ such that for every $\pi \in \Pi$ there is $F \in \mathscr{F}$ such that for each $\lambda \in F$ there exists a positive integer $k_{\lambda}$ with $\rho\left(m\left(C_{k}\right),\left(m_{\lambda}\left(C_{k}\right)\right)\right.$ for any $k \geq k_{\lambda}$.

(iv) For every increasing sequence $\left(C_{k}\right)_{k}$ in $\Sigma$ there is a family $\left(Y_{\pi}\right)_{\pi} \in \mathscr{U}$ such that for each $\pi \in \Pi$ and $F \in \mathscr{F}$ there are $\lambda_{1}, \ldots, \lambda_{q} \in F$ and $\bar{k} \in \mathbb{N}$ such that for each $k \geq \bar{k}$ there exists $j \in[1, q]$ with $\rho\left(m\left(C_{k}\right), m_{\lambda_{j}}\left(C_{k}\right)\right) \in$ $Y_{\pi}$.

Indeed, it is enough to take

$$
\begin{aligned}
a_{\lambda, \zeta} & =m_{\lambda}\left(C_{k}\right), \\
a_{\zeta} & =m\left(C_{k}\right), \\
a_{\lambda, \xi} & =m_{\lambda}(C), \\
a_{\xi} & =m(C),
\end{aligned}
$$

where $\left(C_{k}\right)_{k}$ is a fixed increasing sequence in $\Sigma$, whose union is C. Conditions (i) of Theorem 6 and (ii) and (iii) of Theorem 9 become conditions (ii), (iii), and (iv) of Theorem 13, respectively.

Remark 14. (a) Observe that results analogous to Theorem 13 hold when the involved set functions $m_{\lambda}, \lambda \in \Lambda$, are $\mathscr{U}$ continuous from above or $\mathcal{U}$-(s)-bounded on $\Sigma$, that is, if $(\mathscr{U}) \lim _{k} \rho\left(m_{\lambda}\left(A_{k}\right), 0\right)=0$ for every disjoint sequence $\left(A_{k}\right)_{k}$ in $\Sigma$.

(b) Note that conditions (ii)-(iv) of Theorem 13 are just satisfied, for example, when $R=Y$ is a Dedekind complete lattice group, $\rho(a, b)=|a-b|, a, b \in \mathbb{R}, \Lambda=\mathbb{N}, \mathscr{F}=$ $\mathscr{F}_{\text {cofin }}$, and $\left(m_{n}\right)_{n}$ is a sequence of $\sigma$-additive positive $R$ valued measures, thanks to the classical limit theorems (see also $[16,34,35])$.

The next step is to give necessary and sufficient conditions for absolute continuity of the limit measure.

Let $v: \Sigma \rightarrow \mathbb{R}_{0}^{+}$be a finitely additive measure. We endow $\Sigma$ with the Fréchet-Nikodým topology generated by the pseudometric $\rho_{\nu}(D, E):=|\nu(D)-\nu(E)|, D, E \in \Sigma$. Pick now $\Xi=\Sigma$, and for each $E \in \Sigma$ let $\mathcal{S}_{E}$ be the filter generated by the base $\mathscr{W}:=\left\{\left\{D \in \Sigma: \rho_{\nu}(D, E)<\eta\right\}: \eta>0\right\}$.

We say that $\left(m_{\lambda}\right)_{\lambda}$ is weakly $(\mathscr{U} \mathscr{F})$ - $\nu$-exhaustive at $E \in \Sigma$ iff there is a family $\left(U_{\pi}\right)_{\pi} \in \mathscr{U}$ (depending on $E$ ) such that for each $\pi \in \Pi$ there is $\eta>0$ such that for every $D \in \Sigma$ with $\rho_{\nu}(D, E)<\eta$ there is a set $F_{D} \in \mathscr{F}$ with $\rho\left(m_{\lambda}(D), m_{\lambda}(E)\right) \epsilon$ $U_{\pi}$ whenever $\lambda \in F$. We say that $\left(m_{\lambda}\right)_{\lambda}$ is weakly $(\mathcal{U} \mathscr{F})-\nu$ exhaustive on $\Sigma$ iff it is weakly $(\mathscr{U} \mathscr{F})-\nu$-exhaustive at every $E \in \Sigma$ with respect to a family $\mathbf{X} \in \mathscr{U}$ independent of $E \in \Sigma$. 
A measure $m: \Sigma \rightarrow R$ is said to be $\mathcal{U}$-v-continuous at $E \in \Sigma$ iff there is a family $\left(U_{\pi}\right)_{\pi} \in \mathcal{U}$ (depending on $E$ ) such that for every $\pi \in \Pi$ there is $\eta>0$ with $\rho(m(D), m(E)) \epsilon$ $U_{\pi}$ whenever $\rho_{\gamma}(D, E)<\eta$. We say that $m$ is globally $\mathcal{U}-\nu$ continuous on $\Sigma$ with respect to $\nu$ iff it is $\mathcal{U}-\nu$-continuous at $E$ with respect to $\nu$ for each $E \in \Sigma$, relative to a family $\mathbf{T} \in \mathcal{U}$, independent of $E \in \Sigma$.

The next result is a consequence of Theorem 11.

Theorem 15. Let $m_{\lambda}: \Sigma \rightarrow R, \lambda \in \Lambda$, be a family of measures $\mathcal{U}$ - $\nu$-continuous at a fixed set $E \in \Sigma$ (resp., globally $\mathcal{U}-\boldsymbol{\nu}$ continuous on $\Sigma$ ) with respect to a family $\mathbf{Z} \in \mathscr{U}$ independent of $\lambda$ and $(\Xi \mathcal{U} \mathscr{F})$-convergent to a measure $m_{0}: \Sigma \rightarrow R$. Then the following are equivalent:

(i) The limit measure $m_{0}$ is $\mathcal{U}$-v-continuous at $E$ (resp., globally $\mathcal{U}$ - $\nu$-continuous on $\Sigma$ ).

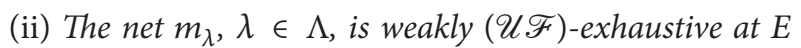
(resp., on $\Sigma$ ).

(iii) There is a family $\left(U_{\pi}\right)_{\pi} \in \mathcal{U}$, depending on $E \in \Sigma$ (resp., independent of $E \in \Sigma)$, such that for each $\pi \in \Pi$ there is $F \in \mathscr{F}$ such that for every $\lambda \in F$ there is $\eta>0$ with $\rho\left(m_{0}(D), m_{\lambda}(D)\right) \in U_{\pi}$ for each $D \in \sum$ with $\rho_{\nu}(D, E)<\eta$.

(iv) There is a family $\left(Y_{\pi}\right)_{\pi} \in \mathcal{U}$, depending on $E \in \Sigma$ (resp., independent of $E \in \Sigma$ ), such that for every $\pi \in \mathbb{N}$ and $F \in \mathscr{F}$ there are $\lambda_{1}, \lambda_{2}, \ldots, \lambda_{q} \in F$ and a positive real number $\eta$ such that for any $D \in \sum$ with $\rho_{\nu}(D, E)<\eta$ there exists $j \in[1, q]$ with $\rho\left(m_{0}(D), m_{\lambda_{j}}(D)\right) \in Y_{\pi}$.

Moreover, if $m_{\lambda}$ 's are globally $\mathcal{U}$-v-continuous, statements (i)(iv) are equivalent to the following:

(v) There is a family $\left(W_{\pi}\right)_{\pi} \in \mathcal{U}$ such that for any $\pi \in \Pi$ and $F \in \mathscr{F}$ there exist a nonempty set $\Lambda_{0} \subset F$ and a finitely uniform cover $\left\{V_{\lambda}: \lambda \in \Lambda_{0}\right\}$ of $\Sigma$ with $\rho\left(m_{0}(D), m_{\lambda}(D)\right) \in W_{\pi}$ whenever $\lambda \in \Lambda_{0}$ and $D \in$ $V_{\lambda}$.

Remark 16. (a) Observe that when $\Lambda=\mathbb{N}, \mathscr{F}=\mathscr{F}_{\text {cofin }}, m_{n}$, $n \in \mathbb{N}$, are positive $\sigma$-additive measures, $R$ is a Dedekind complete lattice group, and $Y=R, \rho(a, b)=|b-a|, a, b \in R$, we get that conditions (ii) $-(\mathrm{v})$ of Theorem 15 are fulfilled, thanks to the limit theorems existing in the literature (see also $[16,34$, 35]).

(b) Let $\Sigma=\mathscr{P}(\mathbb{N})$ be the class of all subsets of $\mathbb{N}$; let $\mathscr{F}$ be a filter containing $\mathscr{F}_{\text {cofin }}$ and $\nu(A)=\sum_{k \in A}\left(1 / 2^{k}\right), A \in \Sigma$. For each $n \in \mathbb{N}$, let us define the Dirac measure $\delta_{n}: \Sigma \rightarrow \mathbb{R}$ by

$$
\delta_{n}(A):= \begin{cases}1, & \text { if } n \in A \\ 0, & \text { if } n \in \mathbb{N} \backslash A\end{cases}
$$

It is not difficult to see that $\delta_{n}$ is $\sigma$-additive on $\Sigma$. Moreover, $\delta_{n}$ is $\nu$-continuous at $\emptyset$ (i.e., $\nu$-absolutely continuous): indeed, if $\vartheta_{n}=1 / 2^{n}$ and $\nu(A)<\vartheta_{n}$, then $n \notin A$, and hence $\delta_{n}(A)=0$. We claim that the sequence $\left(\delta_{n}\right)_{n}$ is not weakly $\mathscr{F}$-exhaustive at $\emptyset$. Indeed, observe that for each $\vartheta>0$ there is a cofinite set $D_{\vartheta} \subset \mathbb{N}$ with $\nu\left(D_{\vartheta}\right)<\vartheta$. Note that since $\mathscr{F}$ contains $\mathscr{F}_{\text {cofin }}$, every element of $\mathscr{F}$ is infinite; otherwise $\emptyset \in \mathscr{F}$, which is impossible. Furthermore, observe that for every infinite subset $F \subset \mathbb{N}$, and a fortiori for any $F \in \mathscr{F}$, there is a sufficiently large integer $\bar{n} \in F \cap D_{\vartheta}$, so that $\delta_{\bar{n}}\left(D_{\vartheta}\right)=1$. From this we deduce that the sequence $\left(\delta_{n}\right)_{n}$ is not weakly $\mathscr{F}$-exhaustive at $\emptyset$. If $\mathscr{F}$ is an ultrafilter of $\mathbb{N}$ containing $\mathscr{F}_{\text {cofin }}$ (the existence of such ultrafilters follows from the Axiom of Choice; see also $[19,36])$, then for every $A \subset \mathbb{N}$ we have

$$
\delta^{\prime}(A):=(\mathscr{F}) \lim _{n} \delta_{n}(A)= \begin{cases}1, & \text { if } A \in \mathscr{F} \\ 0, & \text { if } A \notin \mathscr{F}\end{cases}
$$

We claim that $\delta^{\prime}$ is not $\nu$-continuous at $\emptyset$. Indeed, fix arbitrarily $\eta>0$ and let $\bar{k} \in \mathbb{N}$ be such that $1 / 2^{\bar{k}-1} \leq \eta$. Let $A$ be any element of $\mathscr{F}$ and set $A^{*}:=A \cap\left(\left[\bar{k},+\infty[)\right.\right.$; then $A^{*} \in \mathscr{F}$. We get $\nu\left(A^{*}\right) \leq \sum_{k=\bar{k}}^{\infty}\left(1 / 2^{k}\right)=1 / 2^{\bar{k}-1} \leq \eta$ and $\delta^{\prime}\left(A^{*}\right)=1$, getting the claim.

Furthermore, in this case, conditions (i)-(iv) in Theorem 13 do not hold. Indeed, choose a filter $\mathscr{F}$ of $\mathbb{N}$ containing $\mathscr{F}_{\text {cofin }}$, and let $C_{k}:=[1, k], k \in \mathbb{N}$. Observe that, as said before, every element of $\mathscr{F}$ is infinite. For every $k$ and for any infinite set $F \subset \mathbb{N}$ there is $\bar{n} \in F \backslash C_{k}$, and hence we get $\delta_{\bar{n}}(\mathbb{N})-$ $\delta_{\bar{n}}\left(C_{k}\right)=1$. Thus, in this case, condition (ii) of Theorem 13 is not fulfilled. If $\mathscr{F}$ is an ultrafilter of $\mathbb{N}$, then the measure $\delta^{\prime}$ defined in (22) is not $\sigma$-additive on $\Sigma$. Indeed, if $A$ is any element of $\mathscr{F}$, then we get $\sum_{n \in A} \delta^{\prime}(\{n\})=0$ and $\delta^{\prime}(A)=$ 1.

When $R$ is a Dedekind complete lattice group, $Y=R$, $\rho(a, b)=b-a$, and $\mathcal{U}_{j}^{(0)}, j=1,2,3$, are as in Example 3(c); we obtain some results similar to the previous ones also for semicontinuous set functions (for a related literature, see also [20] and the references therein).

In this setting, the concepts of weak backward (resp., forward) filter exhaustiveness and lower (resp., upper) semicontinuity are formulated as follows.

Definition 17. (a) One says that $\left(m_{\lambda}\right)_{\lambda}$ is weakly $(\mathcal{U} \mathscr{F})-\nu$ backward (resp., forward) exhaustive at $E \in \Sigma$ iff there is a family $\left(U_{\pi}\right)_{\pi} \in \mathscr{U}$ (depending on $E$ ) such that for each $\pi \in \Pi$ there is $\eta>0$ such that for every $D \in \sum$ with $\rho_{\nu}(D, E)<\eta$ there is a set $F_{D} \in \mathscr{F}$ with $m_{\lambda}(E)-m_{\lambda}(D)$ (resp., $m_{\lambda}(D)-$ $\left.m_{\lambda}(E)\right) \in U_{\pi}$ whenever $\lambda \in F$.

(b) One says that $\left(m_{\lambda}\right)_{\lambda}$ is weakly $(\mathcal{U F})$-v-backward (resp., forward) exhaustive on $\Sigma$ iff it is weakly $(\mathscr{U} \mathscr{F})-\nu$ backward (resp., forward) exhaustive at every $E \in \Sigma$ with respect to a family $\mathbf{X} \in \mathcal{U}$ independent of $E \in \Sigma$.

(c) One says that $\left(m_{\lambda}\right)_{\lambda}$ is weakly $(\mathcal{U} \mathscr{F})$ - $\nu$-exhaustive at $E$ (resp., on $\Sigma$ ) iff it is weakly $(\mathscr{U} \mathscr{F})$ - $\nu$-backward and forward exhaustive at $E$ (resp., on $\Sigma$ ).

(d) One says that $m: \Sigma \rightarrow R$ is $\mathcal{U}$-v-lower (resp., upper) semicontinuous at $E \in \Sigma$ iff there is a family $\left(U_{\pi}\right)_{\pi} \in \mathscr{U}$ (depending on $E$ ) such that for every $\pi \in \Pi$ there is $\eta>0$ with $m(E)-m(D)$ (resp., $m(D)-m(E)) \in U_{\pi}$ whenever $\rho_{\nu}(D, E)<$ $\eta$. We say that $m$ is globally $\mathscr{U}$-v-lower (resp., upper) semicontinuous on $\Sigma$ iff it is $\mathcal{U}$ - $\nu$-lower (resp., upper) semicontinuous at $E$ for each $E \in \Sigma$ with respect to a family $\mathbf{T} \in \mathcal{U}$, independent of $E \in \Sigma$. 
Similarly as Theorem 15, it is possible to prove the following result about semicontinuity of the limit set function. The next theorem is given in the case of lower semicontinuity; an analogous result holds in the setting of upper semicontinuity.

Theorem 18. Suppose that $m_{\lambda}: \Sigma \rightarrow R, \lambda \in \Lambda$, are globally $\mathcal{U}$ $\nu$-continuous on $\Sigma$ with respect to a family $\mathbf{S} \in \mathcal{U}$, independent of $\lambda$, and $(\Sigma \mathscr{U} \mathscr{F})$-convergent to a set function $m_{0}: \Sigma \rightarrow R$. Then the following are equivalent:

(i) $m_{0}$ is $\mathscr{U}$-v-lower semicontinuous at $E$ (resp., globally $\mathcal{U}$ $\nu$-lower semicontinuous on $\Sigma$ ).

(ii) The family $\left(m_{\lambda}\right)_{\lambda}$ is weakly $(\mathcal{U F})$-backward exhaustive at $E$ (resp., on $\Sigma$ ).

(iii) There is a family $\left(U_{\pi}\right)_{\pi} \in \mathcal{U}$, depending on $E$ (resp., independent of $E$ ), such that for any $\pi \in \Pi$ there is $F \in \mathscr{F}$ such that for every $\lambda \in F$ there is $\eta>0$ with $m_{\lambda}(D)-m_{0}(D) \in U_{\pi}$ for each $D \in \sum$ with $\rho_{\nu}(D, E)<$ $\eta$.

(iv) There exists a family $\left(V_{\pi}\right)_{\pi} \in \mathcal{U}$, depending on $E$ (resp., independent of $E$ ), such that for every $\pi \in \Pi$ and $F \in$ $\mathscr{F}$ there are $\lambda_{1}, \lambda_{2}, \ldots, \lambda_{q} \in F$ and $\eta>0$ such that for any $D \in \sum$ with $\rho_{\gamma}(D, E)<\eta$ there exists $j \in[1, q]$ with $m_{\lambda_{j}}(D)-m_{0}(D) \in V_{\pi}$.

Moreover, global $\mathcal{U}$-v-lower semicontinuity of $m_{0}$ is equivalent to the following condition:

(v) There is a family $\left(W_{\pi}\right)_{\pi} \in \mathcal{U}$, such that for every $\pi \in \Pi$ and $F \in \mathscr{F}$ there exist a nonempty set $\Lambda_{0} \subset F$ and $a$ finitely uniform cover $\left\{V_{\lambda}: \lambda \in \Lambda_{0}\right\}$ of $\sum$ with $m_{\lambda}(D)-$ $m_{0}(D) \in W_{\pi}$ for all $\lambda \in \Lambda_{0}$ and $D \in V_{\lambda}$.

Remark 19. (a) Let $\mathscr{F}$ be an ultrafilter of $\mathbb{N}$ containing $\mathscr{F}_{\text {cofin }}$, let $v$ be as in Remark 16(b) and let $\delta^{\prime}, \delta_{n}, n \in \mathbb{N}$, be as in (22) and (21), respectively. It is not difficult to check that the sequence $\left(\delta_{n}\right)_{n}$ is weakly $(\mathscr{F})$ - $\nu$-backward exhaustive but not weakly $(\mathscr{F})-\nu$-forward exhaustive at $\emptyset$ and that $\delta^{\prime}$ is $\nu$-lower semicontinuous but not $\nu$-upper semicontinuous at $\emptyset$.

(b) With the same techniques as above, it is possible to prove similar results even when the involved set $\Xi$ is endowed with a bornology, extending earlier theorems proved in $[1,6]$. A bornology on $\Xi$ is a family $\mathscr{B}$ of nonempty subsets of $\Xi$, which covers $\Xi$, stable under finite unions and with $B^{\prime} \in \mathscr{B}$ whenever $\emptyset \neq B^{\prime} \subset B$ and $B \in \mathscr{B}$. Examples of bornologies on $\Xi$ are the classes of all finite nonempty subsets and of all nonempty subsets of $\Xi$, the collection of all nonempty subsets of $\Xi$ with compact closure when $\Xi$ is a topological space, and, if $(\Xi, \rho)$ is a metric space, the families of all nonempty $\rho$ bounded subsets of $\Xi$ and of all nonempty $\rho$-totally bounded subsets of $\Xi$ (see also $[1,14,15]$ and the literature therein).

\section{Conclusions}

We studied the problem of finding conditions for preserving continuity or semicontinuity of the limit family of a doubleindexed family of elements of a set endowed with an abstract structure of convergence.
Our axiomatic approach includes symmetric and asymmetric distance functions, topological and lattice groups, cone (quasi)metric spaces, functions and measures, and nets and filters.

We proved some theorems on exchange of limits, giving some necessary and sufficient conditions in terms of weak filter exhaustiveness, Alexandroff, Arzelà, and strong uniform convergence. As a consequence, we proved some necessary and sufficient conditions for continuity from above/below and absolute continuity of the limit set function of a converging family. We showed that, different from the classical cases, these conditions are not always fulfilled.

Open Problems. (a) Prove some similar results in some other abstract contexts and with respect to other types of convergence.

(b) Investigate some other properties of continuous or semicontinuous functions/measures in abstract settings.

\section{Competing Interests}

The authors declare that they have no competing interests.

\section{Acknowledgments}

This work was supported by Universities of Perugia and Athens and the Italian National Group of Mathematical Analysis, Probability and Applications (GNAMPA).

\section{References}

[1] G. Beer, "The Alexandroff property and the preservation of strong uniform continuity," Applied General Topology, vol. 11, no. 2, pp. 117-133, 2010.

[2] G. Beer, "Semicontinuous limits of nets of continuous functions," Mathematical Programming, vol. 139, no. 1-2, pp. 71-79, 2013.

[3] A. Boccuto and X. Dimitriou, "Ascoli-type theorems in cone metric space setting," Journal of Inequalities and Applications, vol. 2014, p. 420, 2014.

[4] A. Boccuto and X. Dimitriou, "Semicontinuous filter limits of nets of lattice group-valued functions," in Proceedings of the 31st Conference of the Hellenic Mathematical Society, pp. 1-10, Veria, Greece, November 2014.

[5] A. Boccuto and X. Dimitriou, "Asymmetric Ascoli-type theorems and filter exhaustiveness," Applied Mathematical Sciences, vol. 9, no. 108, pp. 5389-5398, 2014.

[6] A. Boccuto and X. Dimitriou, "Strong uniform continuity and filter exhaustiveness of nets of cone metric space-valued functions," in Proceedings of the International Conference on Topology and Its Applications, pp. 21-36, 2015.

[7] A. Boccuto and X. Dimitriou, "On Filter $\alpha$-convergence and exhaustiveness of function nets in lattice groups and applications," Journal of Mathematics Research, vol. 7, no. 2, pp. 56-68, 2015.

[8] A. Caserta, "Decomposition of topologies which characterize the upper and lower semicontinuous limits of functions," Abstract and Applied Analysis, vol. 2011, Article ID 857278, 9 pages, 2011. 
[9] A. Caserta, G. Di Maio, and L. Holá, “(Strong) weak exhaustiveness and (strong uniform) continuity," Filomat, vol. 24, no. 4, pp. 63-75, 2010.

[10] J. Collins and J. Zimmer, "An asymmetric Arzelá-Ascoli theorem," Topology and Its Applications, vol. 154, no. 11, pp. 23122322, 2007.

[11] H.-P. A. Künzi, "Nonsymmetric distances and their associated topologies: about the origins of basic ideas in the area of asymmetric topology," in Handbook of the History of General Topology, C. E. Aull and R. Lowen, Eds., vol. 3, pp. 853-968, 2001.

[12] A. C. Mennucci, "On asymmetric distances," Analysis and Geometry in Metric Spaces, vol. 1, pp. 200-231, 2013.

[13] A. C. Mennucci, "Geodesics in asymmetric metric spaces," Analysis and Geometry in Metric Spaces, vol. 2, pp. 115-153, 2014.

[14] G. Beer, A. Caserta, G. Di Maio, and R. Lucchetti, "Convergence of partial maps," Journal of Mathematical Analysis and Applications, vol. 419, no. 2, pp. 1274-1289, 2014.

[15] G. Beer and S. Levi, "Strong uniform continuity," Journal of Mathematical Analysis and Applications, vol. 350, no. 2, pp. 568589, 2009.

[16] A. Boccuto and X. Dimitriou, Convergence Theorems for Lattice Group-Valued Measures, Bentham Science Publishers, Sharjah, UAE, 2015.

[17] S. Dolecki, "An initiation into convergence theory, beyond topology," in Contemporary Mathematics, vol. 486, pp. 115-161, American Mathematical Society, Providence, RI, USA, 2009.

[18] F. Mynard, "A convergence-theoretic viewpoint on the ArzeláAscoli theorem," Real Analysis Exchange, vol. 38, no. 2, pp. 431444, 2013.

[19] E. Schechter, Handbook of Analysis and Its Foundations, Academic Press, New York, NY, USA, 1997.

[20] Z. Wang and G. J. Klir, Generalized Measure Theory, Springer, New York, NY, USA, 2009.

[21] C. Bardaro, A. Boccuto, X. Dimitriou, and I. Mantellini, "Abstract Korovkin-type theorems in modular spaces and applications," Central European Journal of Mathematics, vol. 11, no. 10, pp. 1774-1784, 2013.

[22] A. Boccuto and X. Dimitriou, "Korovkin-type theorems for abstract modular convergence," Results in Mathematics, vol. 69, no. 3-4, pp. 477-495, 2016.

[23] A. Boccuto, B. Riečan, and M. Vrábelová, Kurzweil-Henstock Integral in Riesz Spaces, Bentham Science Publishers, Sharjah, UAE, 2009.

[24] D. Candeloro and A. R. Sambucini, "Filter convergence and decompositions for vector lattice-valued measures," Mediterranean Journal of Mathematics, vol. 12, no. 3, pp. 621-637, 2015.

[25] D. H. Fremlin, "A direct proof of the Matthes-Wright integral extension theorem," Journal of the London Mathematical Society, vol. 2-11, no. 3, pp. 276-284, 1975.

[26] A. Boccuto and X. Dimitriou, "Matrix theorems and interchange for lattice group-valued series in the filter convergence setting," Bulletin of the Hellenic Mathematical Society, In press.

[27] B. Riečan and T. Neubrunn, Integral, Measure, and Ordering, vol. 411 of Mathematics and Its Applications, Kluwer Academic Publishers, Dordrecht, The Netherlands; Ister Science, Bratislava, Slovakia, 1997.

[28] P. D. Proinov, "A unified theory of cone metric spaces and its applications to the fixed point theory," Fixed Point Theory and Applications, vol. 2013, article 103, 38 pages, 2013.
[29] D. Candeloro, "On the Vitali-Hahn-Saks, Dieudonné and Nikodým theorems," Supplemento ai Rendiconti del Circolo Matematico di Palermo, Serie II, vol. 8, pp. 439-445, 1985 (Italian).

[30] A. Boccuto and X. Dimitriou, "Ideal limit theorems and their equivalence in (l)-group setting," Journal of Mathematics Research, vol. 5, no. 2, pp. 43-60, 2013.

[31] P. S. Alexandroff, Einführung in die Mengenlehre und die Theorie der reellen Funktionen, Deutscher Verlag der Wissenschaften, Berlin, Germany, 1964.

[32] C. Arzelà, "On continuity of the sum of infinitely many continuous functions," Rendiconti dell'Accademia delle Scienze dell'Istituto di Bologna, pp. 79-84, 1883-1884 (Italian).

[33] C. Swartz, Infinite Matrices and the Gliding Hump, World Scientific Publishing, Singapore, 1996.

[34] A. Boccuto and D. Candeloro, "Uniform s-boundedness and convergence results for measures with values in complete Igroups," Journal of Mathematical Analysis and Applications, vol. 265, no. 1, pp. 170-194, 2002.

[35] A. Boccuto and D. Candeloro, "Some new results about BrooksJewett and Dieudonné-type theorems in (1)-groups," Kybernetika, vol. 46, no. 6, pp. 1049-1060, 2010.

[36] W. W. Comfort and S. Negrepontis, The Theory of Ultrafilters, Springer, New York, NY, USA, 1974. 


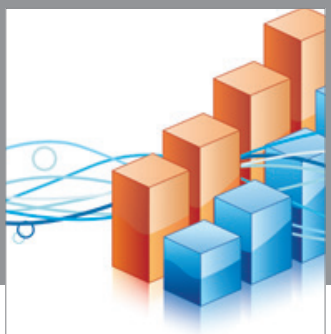

Advances in

Operations Research

vatem alat4

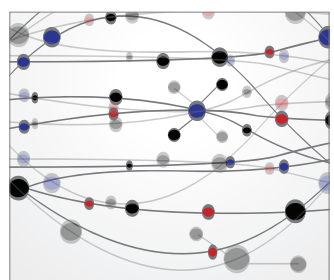

\section{The Scientific} World Journal
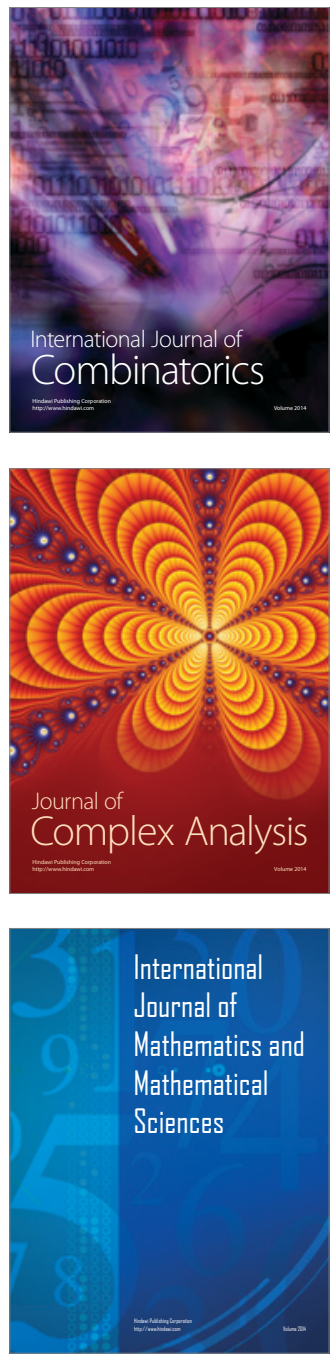
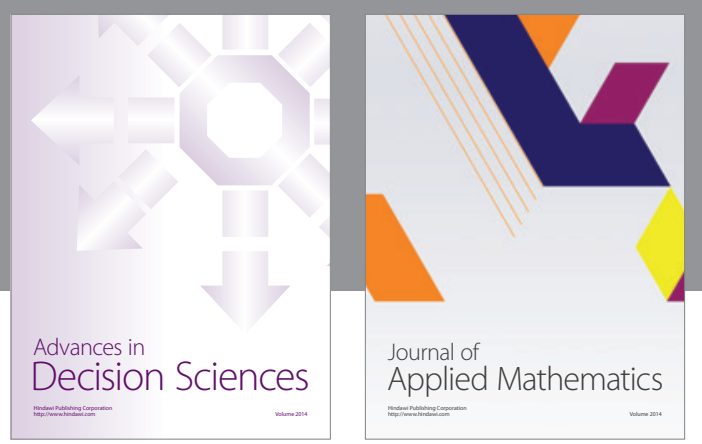

Algebra

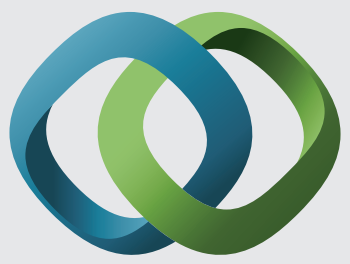

\section{Hindawi}

Submit your manuscripts at

http://www.hindawi.com
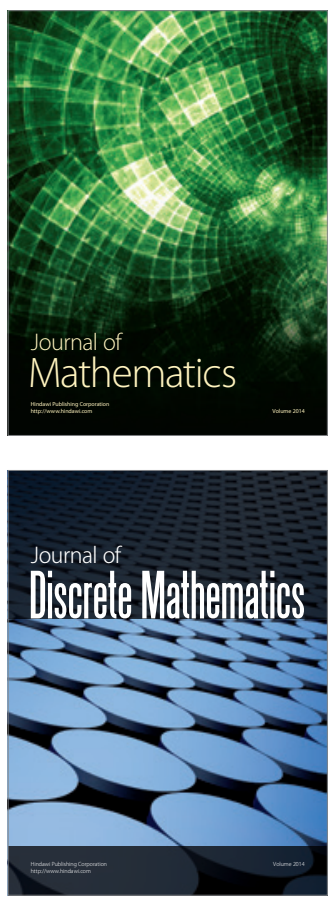

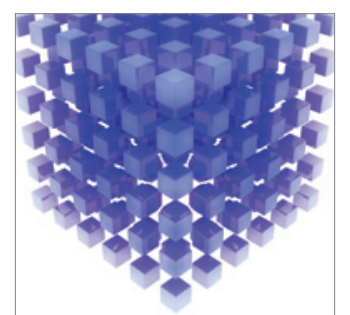

Mathematical Problems in Engineering
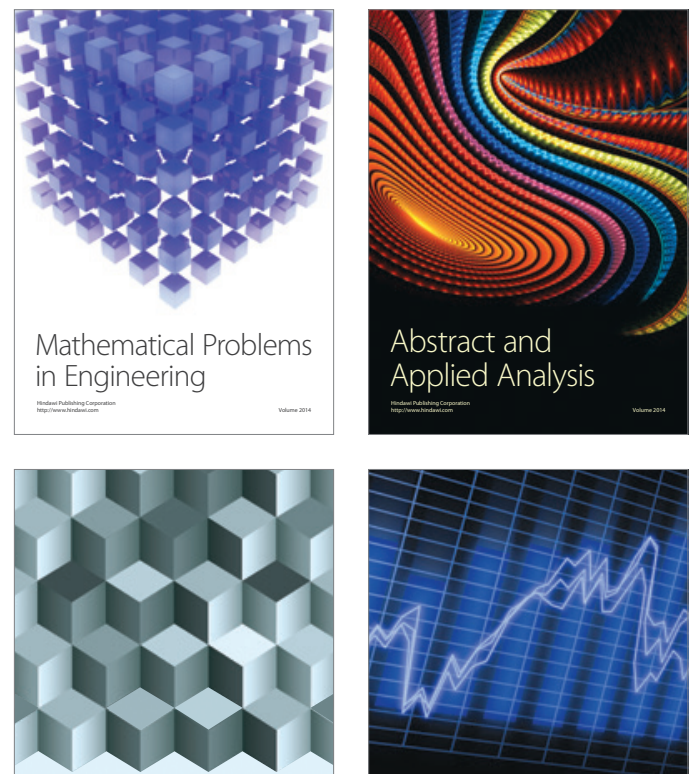

Journal of

Function Spaces

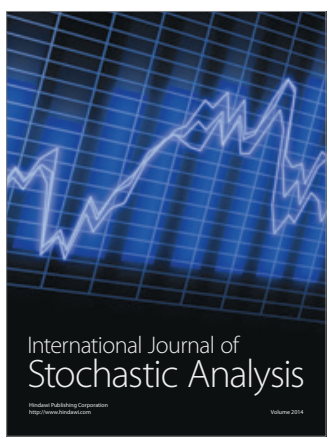

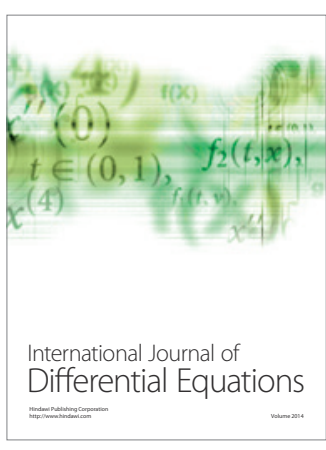
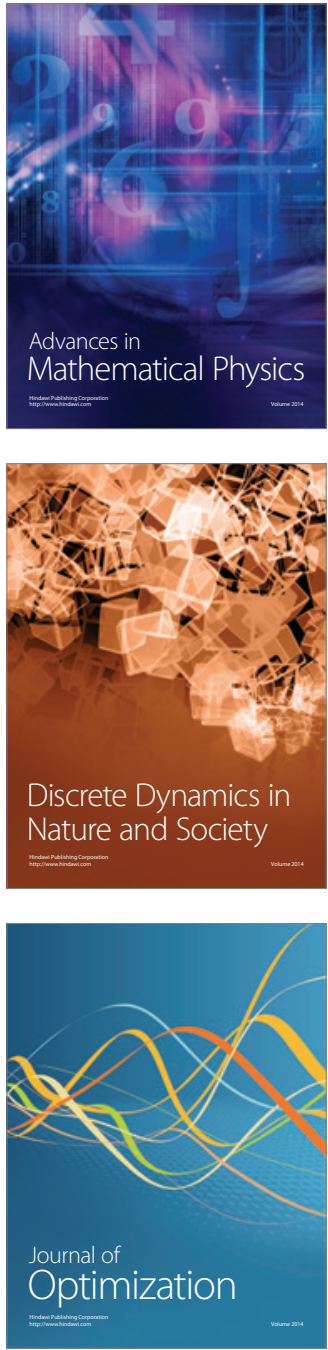